\title{
(2) OPEN ACCESS \\ Different responses of the oral, nasal and lung microbiomes to cigarette smoke
}

\author{
Stefan Pfeiffer, ${ }^{1,2}$ Christian Herzmann, ${ }^{3,4}$ Karoline I Gaede, ${ }^{5,6}$ Draginja Kovacevic, ${ }^{6,7}$ \\ Susanne Krauss-Etschmann, ${ }^{6,7,8}$ Michael Schloter ${ }^{1,2}$
}

- Additional supplemental material is published online only. To view, please visit the journal online (http://dx.doi. org/10.1136/thoraxjnl-2020 216153)

For numbered affiliations see end of article.

\section{Correspondence to}

Dr Susanne Krauss-Etschmann, Early Life Origins of Chronic Lung Disease, Research Center Borstel - Leibniz Lung Center, Borstel D-23845, SchleswigHolstein, Germany;

skrauss-etschmann@fz-borstel. de

Received 7 September 2020 Accepted 19 May 2021

Check for updates

(c) Author(s) (or their employer(s)) 2021. Re-use permitted under CC BY-NC. No commercial re-use. See rights and permissions. Published by BMJ.

To cite: Pfeiffer $S$,

Herzmann C, Gaede Kl, et al. Thorax Epub ahead of print: [please include Day Month

Year]. doi:10.1136/

thoraxinl-2020-216153

\section{ABSTRACT}

To examine the role of smoking on the bacterial community composition of the upper and the lower respiratory tract, a monocentric, controlled prospective study was performed, including healthy smokers, ex-smokers and never-smokers. Smokers were further grouped according to their smoking history. Bacterial diversity was analysed using a molecular barcoding approach based on directly extracted DNA. Our study shows for the first time distinct bacterial response patterns in the upper and lower respiratory tract to cigarette smoking leading to a higher abundance of opportunistic pathogens. The clinical significance of these dysbioses for health needs to be further explored.

The airway microbiome is in proximity to the apical side of respiratory epithelia and thus forms an intimate interface between the epithelial barrier and the external environment. ${ }^{1}$ It has been proposed that smoking modulates the airway microbiome already in healthy individuals, ${ }^{1}$ which then might increase the susceptibility to develop respiratory disease. ${ }^{2}$ So far, cigarette smoking has been linked either to microbial changes of the oral and nasal cavities or to that of the lower respiratory tract of healthy individuals. In the present study, we investigated bacterial diversity patterns along the entire respiratory tract, using nasal and oropharyngeal swabs and bronchoalveolar lavage from two cohorts of healthy never-smokers, ex-smokers and current smokers.

Subjects from two prospective monocentric cohorts were investigated (online supplemental material S1). Smokers were further stratified: long-term smokers were defined by $\geq 10$ cigarettes per day and $\geq 10$ pack-years. Short-term smokers differed from long-term smokers by a shorter smoking history: $\geq 10$ daily cigarettes, but $<10$ pack-years. Mild smokers consumed $<10$ daily cigarettes and had $<5$ pack-years. We enrolled 58 participants. Standardised sampling and processing included deep bilateral nasal swabs $(\mathrm{n}=46)$, bilateral oropharyngeal swabs $(\mathrm{n}=54)$ and bronchoalveolar lavage $(\mathrm{n}=52)$. Active/passive smoking exposure was categorised based on nicotine levels and its metabolites cotinine, 3-hydroxy-cotinine and anabasine, detected by liquid chromatographytandem mass spectrometry in urine (online supplemental material table S2.1). Microbiome analysis included DNA extraction, 16S rRNA gene amplification, library preparation, sequencing and subsequent bioinformatics analysis (online supplemental material S2).

Independent from the smoking status, samples from oropharynx and lungs had a similar bacterial community structure, with Prevotella, Veillonella, Streptococcus and Actinomyces being highly abundant. These four genera were also present in nasal samples, but here Corynebacterium and Staphylococcus were dominant, accounting for $47.1 \%$ of the obtained bacterial reads (online supplemental material tables S2.2 and S2.3). When assessing smoking habits, we found that the relative abundance of Corynebacterium and Dolosigranulum for nasal samples correlated negatively with the maximum number of cigarettes smoked per day $(\mathrm{r}=-0.31, \mathrm{p}=0.06$, and $\mathrm{r}=-0.59, \mathrm{p}=0.07$, respectively). Their relative abundance also negatively correlated with smoking years $(\mathrm{r}=-0.38, \mathrm{p}=0.01$, and $\mathrm{r}=-0.42, \mathrm{p}=0.18$, respectively) and packyears $(\mathrm{r}=-0.44, \mathrm{p}=0.005$, and $\mathrm{r}=-0.40, \mathrm{p}=0.24$, respectively) (figure 1A). However, the number of subjects became very small in this, and the following subanalyses, which is why these results have to be taken with caution. Staphylococcus was positively associated with these three smoking factors (figure 1A), which was mainly due to Staphylococcus epidermidis. The latter was present in all nasal samples but was significantly more abundant in samples from long-term and short-term smokers compared with never-smokers ( $\mathrm{p}<0.05$; figure $1 \mathrm{~B})$. Staphylococcus aureus, although of high relative abundance, was only detected in 15 of 48 participants and did not correlate with the smoking status. Nasal samples showed constant and exclusive presence of Propionibacterium sp, which correlated positively with smoking years $(\mathrm{r}=0.48, \mathrm{p}=0.002$; figure 1A). Many samples of never-smokers and mild smokers had a high relative abundance of Dolosigranulum pigrum in contrast with short-term and long-term smokers $(p<0.05$; online supplemental material figure S2.2A). This strain negatively correlated with all smoking-related parameters. Instead, the relative abundance of Prevotella correlated positively with maximum of cigarettes per day $(r=0.041, p=0.004$; figure 1A).

For oropharyngeal samples, Firmicutes had a higher relative abundance in smokers compared with never-smokers $(\mathrm{p}<0.05$, figure $2 \mathrm{~B})$. Although, the differences of its most common genera Streptococcus and Veillonella were neither associated with smoking intensity nor duration. Among Actinobacteria, the significantly higher abundance in smokers and ex-smokers compared with neversmokers ( $\mathrm{p}<0.05$; Figure $3 \mathrm{~B}$ ) was mainly explained by the higher abundance of Actinomyces (neversmokers vs mild smokers: $\mathrm{p}=0.005)$ and Atopobium (never-smokers vs smokers: $\mathrm{p}<0.05$; online supplemental material figure S2.2B). This genus 
A
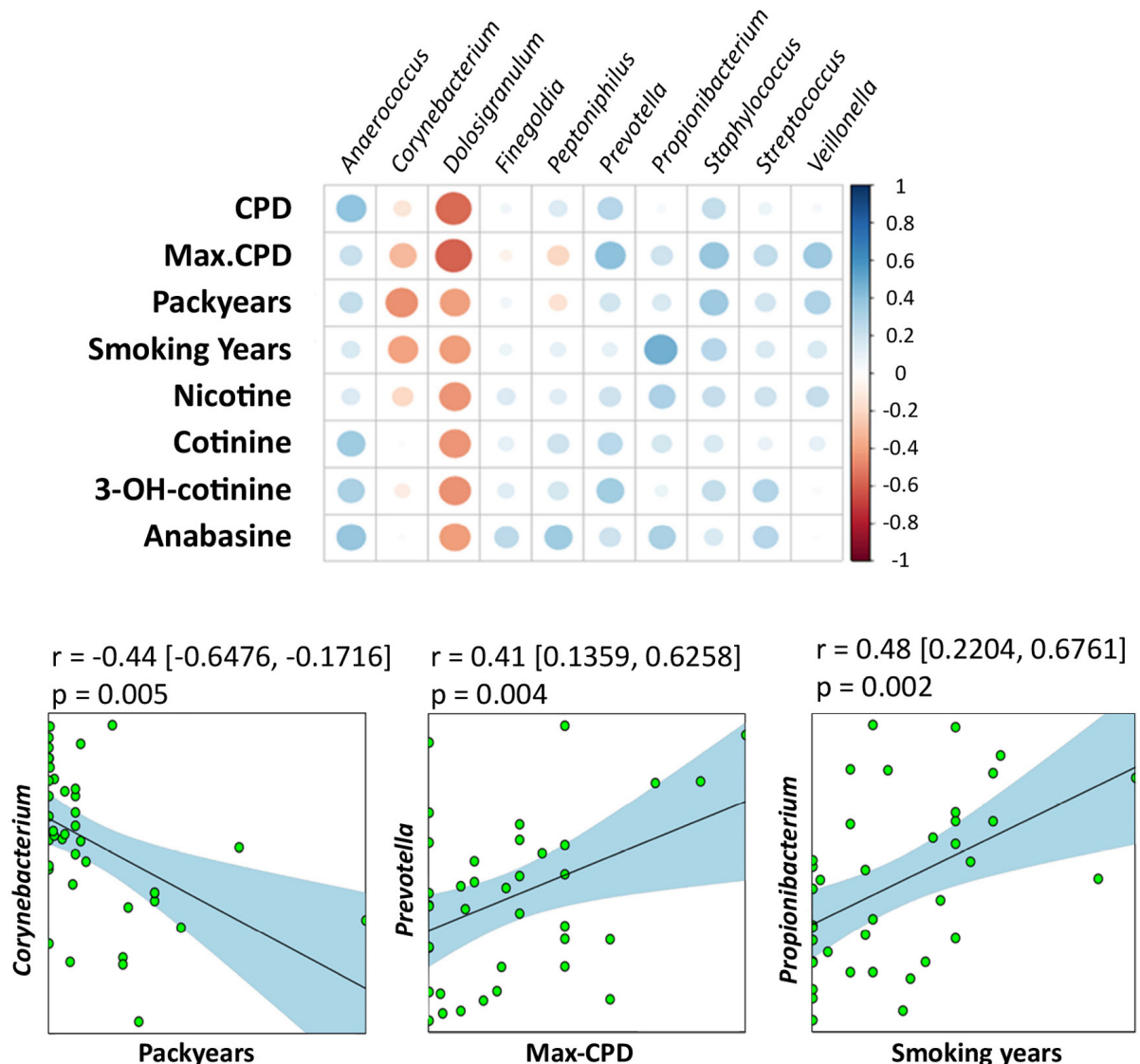

Max-CPD

Smoking years

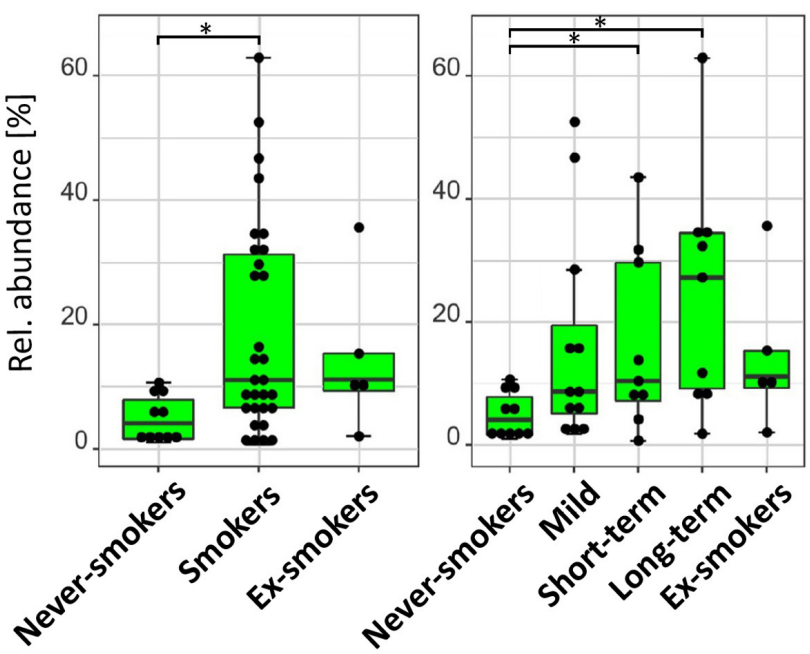

Figure 1 Influence of cigarette smoke on the nasal microbiota. (A) Upper panel: Correlation array of smoking factors with nasal cavity ( $\mathrm{n=46)}$ genera across smoking parameters and transformed sequencing data (centred log-ratio transformation) to remove constrains introduced by the compositionality of the data. The size of the circles shows the significance of the correlation (bigger circle corresponds to a lower $p$ value). The colour of the circle indicates the Pearson correlation between 1 (blue, positive correlation) and -1 (red, negative correlation) between taxa (horizontal) and smoking parameters (vertical). Lower panel: Pearson correlation of bacterial taxa with smoking-related parameters. Dots represent samples. The line corresponds to the fitted linearmodel. Cls of the model are shown in square brackets and by the light blue area. (B) Boxplots show median relative abundances of zero-radius operational taxonomic units of Staphylococcus epidermidis among smoking groups. Smoking status: never-smokers ( $\mathrm{n}=10)$, ex-smokers $(n=6)$ and smokers $(n=30)$; smoking history: heavy long-term smokers (long-term: $n=9)$, heavy smokers (short-term: $n=9)$ and mild/ occasional smokers (mild: $n=12$ ). Significant differences in relative abundance were determined using pairwise Mann-Whitney $U$ test $(p<0.05$, FDR corrected) and are indicated by horizontal brackets with * $(p<0.05)$. CPD, cigarettes per day; Max-CPD, maximum cigarettes per day. 
correlated positively with cotinine $(r=0.41, p=0.002)$ and cigarettes per day $(r=0.32, p=0.02)$, but not with long-term smoking (figure $3 \mathrm{~B}$ ). In contrast to Firmicutes and Actinobacteria, the relative abundance of Betaproteobacteria was lower in the oropharynx from smokers and ex-smokers compared with never-smokers $(p<0.05)$. Likewise, the relative abundance of fusobacterial genus Leptotrichia and Firmicutes strain Centipeda periodontii correlated negatively with cotinine and short-term smoking parameters (figure $3 \mathrm{~A}$ ).

The effect of smoking on the relative abundances of Bacteroidetes and Firmicutes, and the proteobacterial genus Neisseria $(\mathrm{p}<0.05$; online supplemental figure S2.2B), in oropharyngeal

A
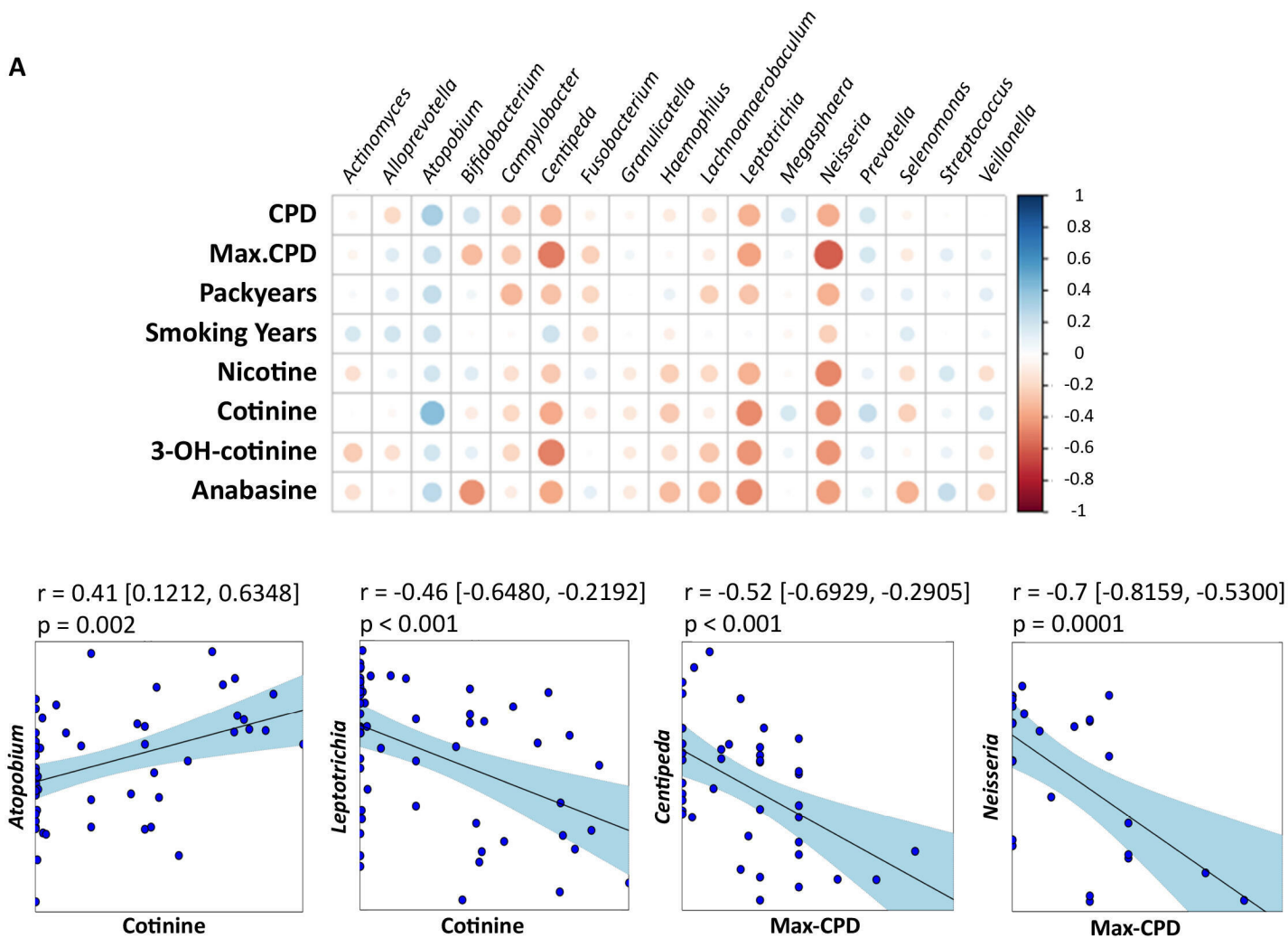

B
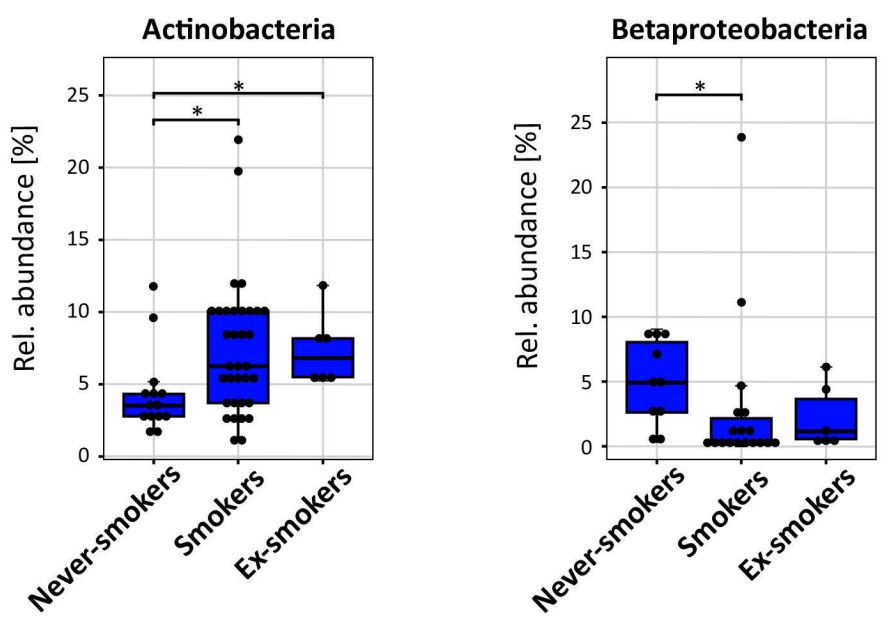

Figure 2 Influence of cigarette smoke on the oropharyngeal microbiota. (A) Upper panel: correlation array of smoking factors with oropharynx $(n=54)$ genera across smoking parameters and transformed sequencing data (centred log-ratio transformation) to remove constrains introduced by the compositionality of the data. The size of the circles shows the significance of the correlation (bigger circle corresponds to a lower $p$ value). The colour of the circle indicates the Pearson correlation between 1 (blue, positive correlation) and -1 (negative correlation) between taxa (horizontal) and smoking parameters (vertical). Lower panel: Pearson correlation of bacterial taxa with smoking-related parameters. Dots represent samples. The line corresponds to the fitted linear model. Cls of the model are shown in square brackets and by the light blue area. (B) Boxplots showing significantly different median relative abundances of zero-radius operational taxonomic units related to different taxa among smoking groups. Smoking status: never-smokers $(n=14)$, smokers $(n=34)$ and ex-smokers $(n=6)$ of paired samples. Significant differences in relative abundance were determined using pairwise Mann-Whitney $U$ test $(p<0.05$, false discovery rate-corrected) and are indicated by horizontal brackets with * $(p<0.05)$. $\mathrm{CPD}$, cigarettes per day; Max-CPD, maximum cigarettes per day. 
A
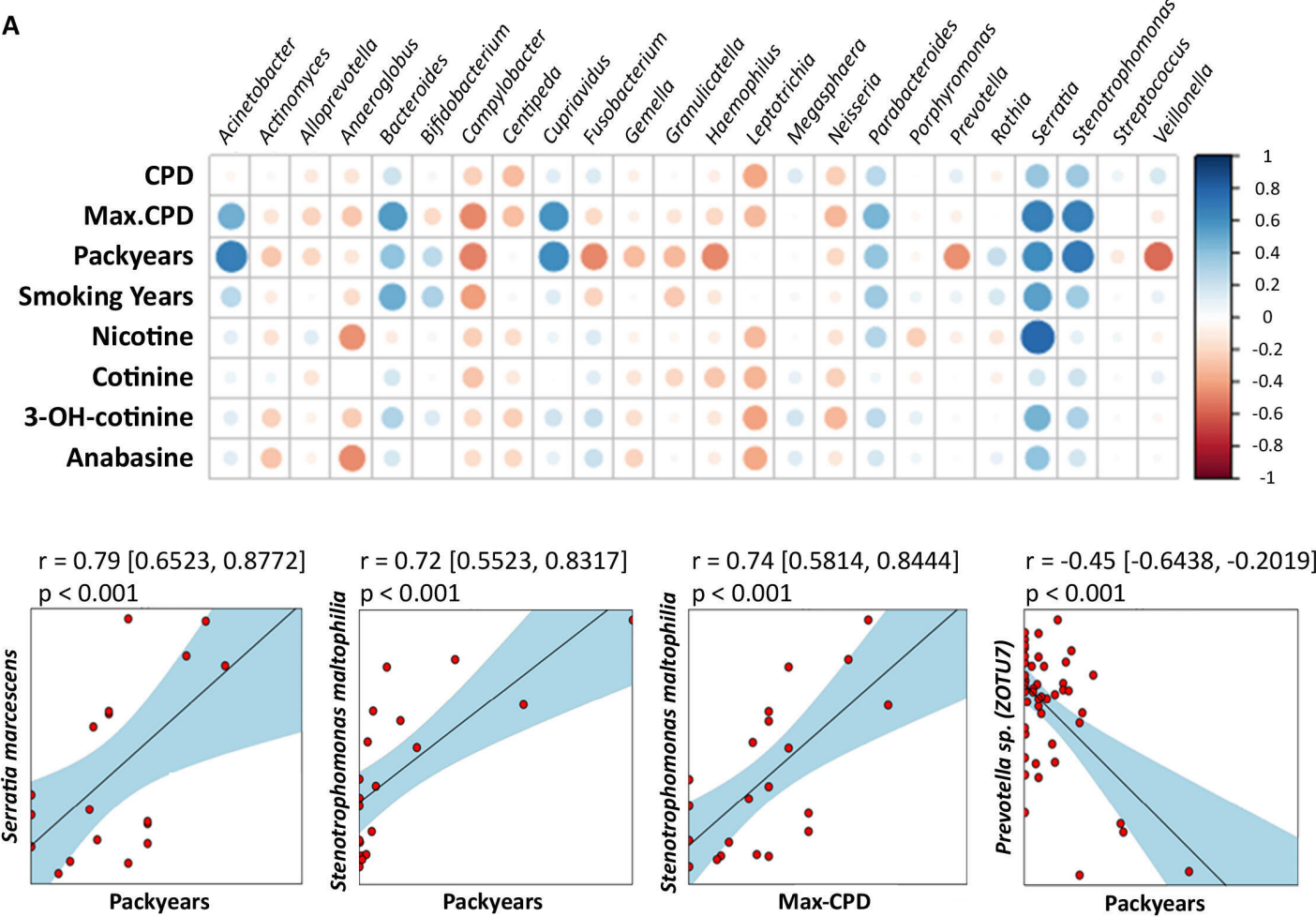

B
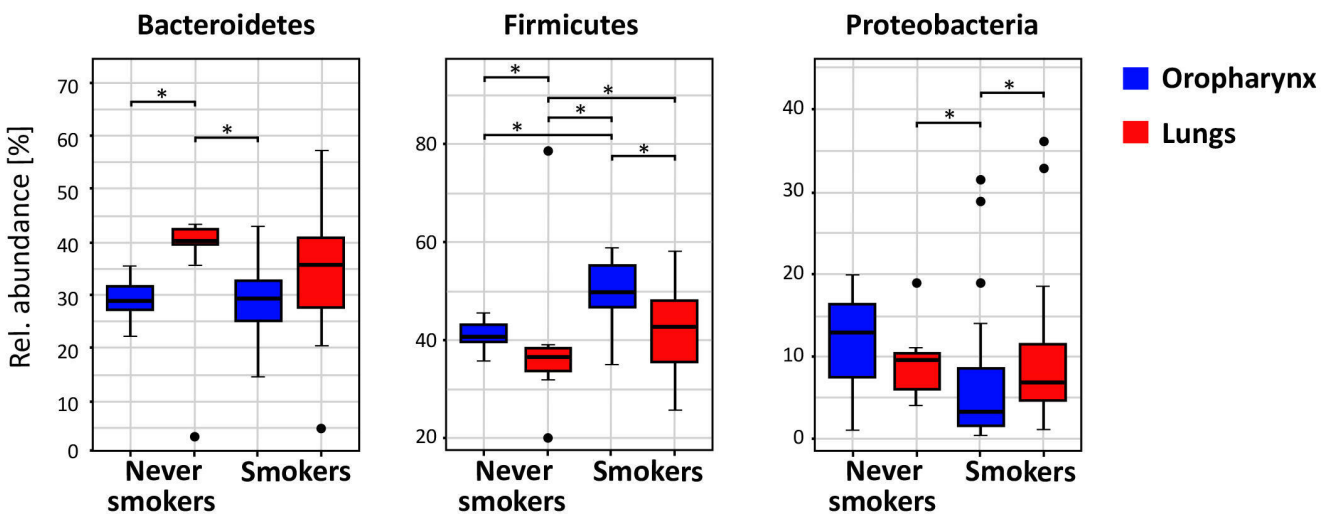

Figure 3 Influence of cigarette smoke on the lung microbiota. (A) Upper panel: correlation array of smoking factors with lungs ( $\mathrm{n}=52$ ) genera across smoking parameters and transformed sequencing data (centred log-ratio transformation) to remove constrains introduced by the compositionality of the data. The size of the circles shows the significance of the correlation (bigger circle corresponds to a lower $p$ value). The colour of the circle indicates the Pearson correlation between 1 (blue, positive correlation) and -1 (red, negative correlation) between taxa (horizontal) and smoking parameters (vertical). Lower panel: Pearson correlation of bacterial taxa with smoking-related parameters. Dots represent samples. The line corresponds to the fitted linear model. Cls of the model are shown in square brackets and by the light blue area. The correlation coefficient (upper row), the false discovery rate-corrected $p$ value (middle row) and the number of observations (lower row) are shown in the corners of the plots. (B) Boxplots showing significantly different median relative abundances of the three most abundant phyla between oropharyngeal and lung samples of never-smokers and smokers. Brackets with * indicate significant $(p<0.05$, pairwise Mann-Whitney $U$ test, Benjamin Hochberg corrected) differences between sample group. CPD, cigarettes per day; Max-CPD, maximum cigarettes per day.

samples was also seen in lung samples (figure $2 \mathrm{~B}$ and online supplemental figure S2.2C). In contrast, several $\gamma$-proteobacterial and $\beta$-proteobacterial species were specific for the microbial composition of lung samples (online supplemental material S3) and were further positively correlated with all smoking-related parameters. Specifically, Acinetobacter bereziniae, Acinetobacter johnsonii, Cupriavidus metallidurans, Serratia marcescens and Stenotrophomonas maltophilia were strongly influenced both by long-term smoking and smoking intensity (figure 2A). Aside from Proteobacteria, also lung-specific Bacteroidetes correlated positively with long-term smoking. Contrarily, the relative abundance of species related to the genera Prevotella $(r=-0.45$, $\mathrm{p}=0.001)$ and Veillonella $(\mathrm{r}=-0.55, \mathrm{p}<0.001)$ negatively correlated to pack-years in lung samples (figure $2 \mathrm{~A}$ ).

Our study reveals for the first time how individual smoking histories affect the bacterial community composition simultaneously in the upper and lower respiratory tract of healthy individuals. We revealed the impact of smoking on the relative abundances of distinct potential pathogenic bacterial groups in different areas of the respiratory system. 
Anaerobic and facultative anaerobic Actinobacteria and Firmicutes like Streptococcus and Veillonella could outgrow aerobic Proteobacteria such as Neisseria due to the depletion of oxygen in the oropharyngeal microbiome. ${ }^{3}$ Microaspiration from the oropharynx is a major source of microbes for the lungs and shapes the pulmonary bacterial community composition. ${ }^{4}$ We confirmed the strong overlap of the oropharyngeal and lung microbiota, but this dynamic relationship was obviously disturbed by cigarette smoke. While the relative abundance of Proteobacteria was lower in smokers compared with never-smokers in both sampling compartments, among smokers that relative abundance was higher in the lungs. As cigarette smoke injures lung epithelia, the likelihood of bacterial colonisation by strong biofilm formers is increased. ${ }^{5}$ Thus, Betaproteobacteria and Gammaproteobacteria, which we found exclusively in the lungs, might be colonisers of injured airways. As mentioned earlier, due to small size of subgroups, these statements should be taken with caution. Furthermore, we observed that the abundance of these taxa was clearly linked to each other and correlated strongly with smoking intensity and duration. Additionally, these taxa are capable of biofilm formation and are potential pathogens. For example, S. maltophilia, a proposed biomarker for cystic fibrosis disease severity, is an important, often multidrug-resistant opportunistic pathogen due to its biofilm-forming capability and its ability to produce antibiotic-inhibiting beta-lactamases. ${ }^{6}$ Serratia marcescens, a potential pathogen resistant to several antibiotics, can grow on cigarette tobacco, and might be transported into the lungs via tobacco flakes. ${ }^{7}$ C. metallidurans can cause secondary infection in immune-compromised individuals and is associated with colonisation of lung lesions. ${ }^{8}$ A. bereziniae has been associated with skin diseases and mucosal infections. ${ }^{9}$ The parallel decrease of Prevotella sp, which is suggested to play a key role in central homeostatic processes, ${ }^{10}$ could further indicate a dysbiosis of the lung microbiome, which is driven by the severity and duration of smoking exposure. Of note, microbiome data are limited to largely descriptive analyses, and, therefore, it is hard to clearly conclude to what extent changes in microbial structure are related to clinical outcomes. Although the age range differs among groups, a larger study is needed to account for potential confounder.

\section{Author affiliations}

${ }^{1}$ Technical University of Munich ZIEL - Institute for Food and Health, Freising, Germany

${ }^{2}$ Research Unit of Comparative Microbiome Analysis, Helmholtz Center Munich German Research Center for Environmental Health, Neuherberg, Germany ${ }^{3}$ Center for Clinical Studies, Research Center Borstel - Leibniz Lung Center, Borstel, Germany

${ }^{4}$ Clinical Trials Unit, German Center for Infection Research (DZIF), Borstel, Germany ${ }^{5}$ BioMaterialBank North, Research Center Borstel - Leibniz Lung Center, Borstel, Germany

${ }^{6}$ German Center for Lung Research (DZL), Airway Research Center North (ARCN),
Borstel, Germany

${ }^{7}$ Early Life Origins of Chronic Lung Disease, Research Center Borstel - Leibniz Lung Center, Borstel, Germany

${ }^{8}$ Institute for Experimental Medicine, Christian-Albrechts-Universität zu Kiel, Kiel, Germany

Acknowledgements The authors thank Kevin Korfmann, Cornelia Galonska and Susanne Kublik for their help in library preparation and sequencing. The authors thank Andrea Glaewe, Johanna Döhling, Lenka Krabbe, Vanessa Schümann, Gabi Huss, Birgit Kullmann and Steffi Fox (all Research Centre Borstel) for coordinating and sampling the study participants and collecting clinical data. The BioMaterialBank Nord is supported by the German Centre for Lung Research and member of popgen 2.0 network, which is supported by a grant from the German Ministry for Education and Research (grant number: 01EY1103).

Contributors SP had full access to all study data in and takes responsibility for the data integrity and accuracy of the data analysis. $\mathrm{CH}$ recruited the study subjects and obtained the microbiological samples. CH, KIG, SK-E, DK and MS contributed substantially to the study design and the writing of the manuscript.

Funding Leibniz Competition 2016, 'The lung microbiota at the interface between airway epithelium and environment'.

Competing interests None declared.

Patient consent for publication Not required.

Ethics approval Cohort 1: German Register for Clinical Trials (DRKS00016932). Cohort 2: clinicaltrials.gov (NCT03562442).

Provenance and peer review Not commissioned; externally peer reviewed.

Open access This is an open access article distributed in accordance with the Creative Commons Attribution Non Commercial (CC BY-NC 4.0) license, which permits others to distribute, remix, adapt, build upon this work non-commercially, and license their derivative works on different terms, provided the original work is properly cited, appropriate credit is given, any changes made indicated, and the use is non-commercial. See: http://creativecommons.org/licenses/by-nc/4.0/.

\section{REFERENCES}

1 Dickson RP, Erb-Downward JR, Freeman CM, et al. Bacterial topography of the healthy human lower respiratory tract. mBio 2017;8. doi:10.1128/mBio.02287-16. [Epub ahead of print: 14 Feb 2017]

2 Li K-J, Chen Z-L, Huang Y, et al. Dysbiosis of lower respiratory tract microbiome are associated with inflammation and microbial function variety. Respir Res 2019;20:272.

3 Wu J, Peters BA, Dominianni C, et al. Cigarette smoking and the oral microbiome in a large study of American adults. Isme J 2016;10:2435-46.

4 Dickson RP, Erb-Downward JR, Freeman CM, et al. Bacterial topography of the healthy human lower respiratory tract. mBio 2017;8. doi:10.1128/mBio.02287-16. [Epub ahead of print: 1402 2017].

5 Wu BG, Segal LN. Lung microbiota and its impact on the mucosal immune phenotype. Microbiol Spectr 2017;5.

6 Chawla K, Vishwanath S, Gupta A. Stenotrophomonas maltophilia in lower respiratory tract infections. J Clin Diagn Res 2014;8:DC20-2

7 Pauly JL, Waight JD, Paszkiewicz GM. Tobacco flakes on cigarette filters grow bacteria: a potential health risk to the smoker? Tob Control 2008;17 Suppl 1:i49-52.

8 Zhou Y, Lin F, Cui Z, et al. Correlation between either Cupriavidus or Porphyromonas and primary pulmonary tuberculosis found by analysing the microbiota in patients' bronchoalveolar lavage fluid. PLOS One 2015;10:e0124194.

9 Jain AL, Harding CM, Assani K, et al. Characteristics of invasive Acinetobacter species isolates recovered in a pediatric academic center. BMC Infect Dis 2016;16:346.

10 Bernasconi E, Pattaroni C, Koutsokera A, et al. Airway microbiota determines innate cell inflammatory or tissue remodeling profiles in lung transplantation. Am J Respir Crit Care Med 2016:194:1252-63. 